\title{
Diversidad genética y distribución regional de cepas de Mycobacterium bovis del ganado en México
}

\author{
Genetic diversity and geographic distribution of \\ Mycobacterium bovis from cattle in Mexico
}

\author{
Feliciano Milián Suazo ${ }^{a}$, Leticia García Casanovaa, Cecilia Romero Torres ${ }^{b}$, Germinal J. \\ Cantó Alarcónc ${ }^{c}$ José A Gutiérrez Reyes ${ }^{d}$, Susana Gallegos Sosa ${ }^{a}$, Marcela Mercado Pezzat ${ }^{b}$, \\ Félix Mejía Estrada F.e, Aída L. Peña Cisneros ${ }^{f}$, Ciro Estrada Chávezg Oscar Pizano Martínez ${ }^{\mathrm{a}}$
}

\begin{abstract}
RESUMEN
Se obtuvieron patrones moleculares (espoligotipos) de 878 aislados de Mycobacterium bovis de ganado de diferentes regiones de México entre los años 2009 y 2010. Setenta y dos por ciento (72 \%) de los espoligotipos cayeron en nueve grupos y $27 \%$ de los aislados dentro de sólo dos espoligotipos; 149 fueron espoligotipos individuales. Los dos espoligotipos predominantes, arbitrariamente identificados como SP1 y SP2, se distribuyen en la mayor parte del territorio nacional, en especial en la zona centro de México en ganado especializado en producción de leche. A pesar de la amplia distribución geográfica de los espoligotipos de mayor frecuencia, algunos muestran cierta localización, en especial los encontrados en zonas geográficas distantes, como es el caso de Chihuahua y Baja California. Aunque pocos, algunos espoligotipos muestran patrones moleculares distintos a los mostrados por los espoligotipos de mayor frecuencia, sugiriendo fuentes de infección desconocida. La mayoría de los Estados con ganadería predominantemente lechera muestran espoligotipos comunes, lo que sugiere intercambio regional frecuente de ganado. Algunos espoligotipos son comunes en ganado para leche y ganado para carne, lo que sugiere transmisión entre estas dos poblaciones; se desconoce, sin embargo, si los animales de carne infectados provienen de explotaciones extensivas o si son de engordas ubicadas dentro de la explotación lechera. Se propone la tipificación rutinaria de aislados de $M$. bovis obtenidos en todos los laboratorios de diagnóstico y mejorar la captura de información epidemiológica de los casos, para hacer mejores conclusiones epidemiológicas de la distribución espacial de las cepas de este agente en el territorio nacional.
\end{abstract}

PALABRAS CLAVE: Tuberculosis, Mycobacterium bovis, Epidemiología molecular, Spoligotyping, Tipificación.

\begin{abstract}
The molecular fingerprints of 878 isolates of Mycobacterium bovis from cattle, mostly dairy cattle, collected from cattle between 2009 and 2010 in different regions of Mexico were obtained by spoligotyping. Seventy-two percent (72 \%) of the spoligotypes fell into nine clusters, and $27 \%$ of the isolates fell into only two spoligotypes; 149 were orphan spoligotypes. The two predominant spoligotypes, arbitrarily designated as SP1 and SP2, were found in almost all States in Mexico, especially in central Mexico, where a concentration of dairy cattle is known. In spite of the wide distribution of spoligotypes observed, some show high regional preference, especially those in geographically distant regions. Only a few spoligotypes show patterns completely different from those shown by the most frequent spoligotypes, suggesting strange sources of infection or the formation of new genetic lines derived from non-lethal mutations. Most States with predominantly high dairy cattle populations showed similar spoligotypes, suggesting exchange of animals between regions. Some spoligotypes are common to dairy and beef cattle, suggesting transmission between populations, most probably due to the movement of dairy cattle to non-dairy regions.
\end{abstract}

KEY WORDS: Tuberculosis, Mycobacterium bovis, Molecular epidemiology, Spoligotyping, Genetic typing.

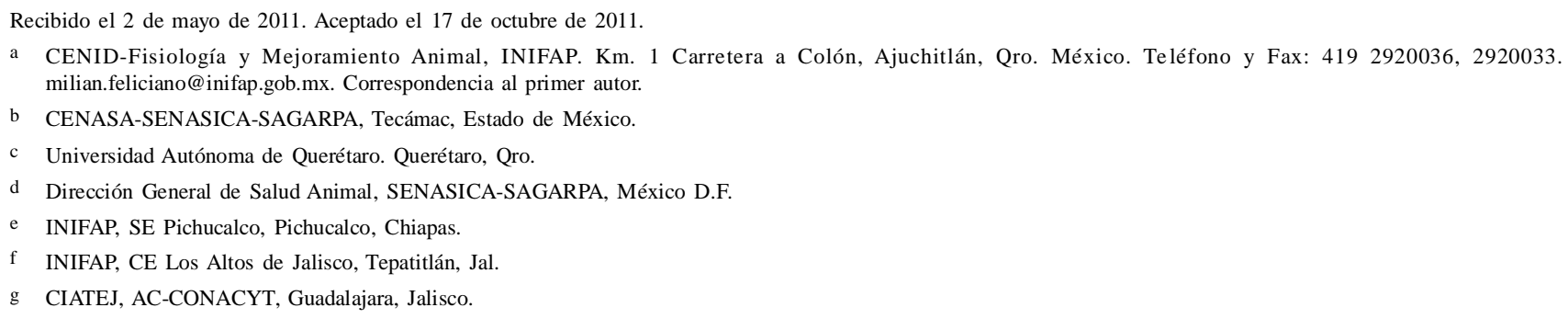




\section{INTRODUCCIÓN}

La tuberculosis bovina (TBb) es causada por el bacilo $M$. bovis, bacteria de crecimiento lento e intracelular obligado(1), de distribución mundial y capaz de infectar a diferentes especies animales $(2)$ $\mathrm{y}$ al hombre $(3,4,5)$. Esta enfermedad causa pérdidas económicas en la ganadería y representa un riesgo para la salud pública(6); la participación de $M$. bovis en humanos en México ha sido estimada en 7 \%(7), mientras que en sur de los Estados Unidos de Norteamérica (USA) llega al $40 \%(8)$. En la última década la $\mathrm{TBb}$ se ha convertido en una barrera no arancelaria para el comercio de ganado $^{(9)}$. Para reducir su impacto, la Dirección General de Sanidad Animal del Servicio Nacional de Sanidad Inocuidad y Calidad Agroalimentaria (SENASICA) ha establecido un Plan Estratégico que contempla a la investigación científica para sustentar actividades de campaña. Uno de estos proyectos está relacionado con la epidemiología molecular (EM) del agente etiológico, M. bovis(10).

Los mecanismos de persistencia y transmisión de la $\mathrm{TBb}$ en las poblaciones es poco entendida(11). Estudios sobre la dinámica de la enfermedad han sido tradicionalmente afectados por diversos factores que impiden la identificación de la relación epidemiológica de los casos. La movilización del ganado, la transmisión entre bovinos dentro y entre hatos, y la participación de la fauna silvestre, son factores que al igual que ocurre en otros países $(12,13)$ en México son importantes, sin embargo, cuantificar el impacto de cada uno es complicado y costoso(14).

La EM es usada para entender mejor el comportamiento de la tuberculosis. Estos estudios se basan en la premisa de que animales infectados con cepas de patrón molecular idéntico son el resultado de transmisión reciente, mientras que aquéllos infectados por cepas de patrón único, se consideran cepas nuevas resultantes de mutaciones no letales $(15)$. Sin embargo, es importante resaltar que patrones moleculares idénticos no necesariamente indican relación epidemiológica, y

\section{INTRODUCTION}

Bovine tuberculosis (BTB) is caused by the bacillus M. bovis, a slow growing and intracellular microorganism(1). M. bovis is able to infect different animal species $(2)$ and men $(3,4,5)$ and is present all over the world. BTB causes economic losses to livestock and represents a risk to public health(6); in Mexico, M. bovis in humans has been estimated in $7 \%(7)$, while in the South of the United States (USA) that proportion is as high as $40 \%(8)$. Lately, BTB has become a non-tariff barrier to the trade of animals and animals' products ${ }^{(9)}$. To reduce the impact of tuberculosis, the Direccion General de Sanidad Animal of the Servicio Nacional de Sanidad Inocuidad y Calidad Agroalimentaria (SENASICA) has established a strategic plan that involves scientific research to support the national campaign. One of these projects is the molecular epidemiology (ME) of the etiological agent, M. bovis(10).

The mechanisms of persistence and transmission of BTB in populations is not well known(11). Studies on the dynamics of the disease have traditionally been affected by various factors impeding the identification of the epidemiological relationship of cases. As it happens in other countries, in Mexico livestock mobilization, transmission among cattle within and between herds, and the participation of wildlife, are important factors $(12,13)$; however, to quantify the impact of each factor is complicated and expensive ${ }^{(14)}$.

$\mathrm{ME}$ is used to better understand tuberculosis performance. ME studies are based on the premises that animals infected with strains of similar molecular pattern are the result of recent transmission, while those infected with strains of unique pattern are new strains resulting from nonlethal mutations ${ }^{(15)}$. However, it is important to highlight that identical patterns do not necessarily indicate epidemiological relationship, and cases epidemiologically linked do not always have the same molecular pattern; therefore, information collected in the routine bases, is essential. 
casos epidemiológicamente ligados no siempre tienen el mismo patrón molecular, lo que hace indispensable sustentar los hallazgos moleculares con información epidemiológica rutinaria.

Uno de los métodos más usados para la tipificación de cepas de $M$. bovis es la espoligotipificación (spoligotyping)(16). Este método ha demostrado su utilidad en una gran cantidad de estudios con una amplia diversidad de objetivos y especies animales(17-23). En comparación con otros métodos, es fácil de realizar, requiere poca cantidad de ADN, es robusto y altamente reproducible, además de que puede ser aplicado a especímenes clínicos(16). Más que eso, spoligotyping es de utilidad en cepas de $M$. bovis con pocas copias del elemento de inserción IS6110, como ocurre con $M$. bovis obtenido del ganado(24). Así, el objetivo del presente trabajo fue identificar los patrones moleculares (espoligotipos) de cepas de M. bovis más frecuentes en las diferentes regiones de México, con el fin de apoyar las actividades de la campaña nacional contra la tuberculosis bovina, y elaborar una base de datos que apoye los programas de control y los acuerdos de comercialización interna y de exportación de ganado en México.

\section{MATERIALES Y MÉTODOS}

\section{Aislados}

Ochocientos setenta y ocho (878) aislados de $M$. bovis fueron obtenidos del ganado en diferentes regiones de México entre el 2009 y el 2010. En un muestreo pasivo por conveniencia y buscando la representación de cada Estado, a cada uno de los Laboratorios Regionales Acreditados para el Diagnóstico de la Tuberculosis Bovina en México se le solicitó, a través de la Dirección General de Salud Animal, el envío de un mínimo de 15 aislados de $M$. bovis de cada uno de los estados de los que tuvieran muestras, incluyendo la hoja de laboratorio con la información epidemiológica correspondiente. Por otro lado, buscando incrementar la muestra, y cubrir los faltantes de Estados no representados o con bajo número de aislados, en un proceso activo se obtuvieron lesiones sospechosas de tuberculosis
The most popular method for $M$. bovis genotyping is spoligotyping $(16)$. This method has proved useful in a large number of studies with a wide range of objectives and animal species(17-23). In comparison with other methods, it is easy to perform, very small amounts of DNA, it is robust and highly reproducible, and it can be applied to clinical specimens $(16)$. More than that, spoligotyping is very useful in strains of $M$. bovis with a few copies of the IS6110 insertion element, as it is the case in strains from cattle (24). Therefore, the objective of this study was to identify molecular patterns (spoligotypes) and distribution of $M$. bovis strains from different regions of Mexico. This, in order to support activities of the national campaign, and to develop a database to support international agreements for trade of animals and animals' products.

\section{MATERIALS AND METHODS}

\section{Isolates}

Eight hundred and seventy-eight (878) isolates of M. bovis were obtained between 2009 and 2010 from cattle in different regions of Mexico were included in the study. In a convenience sampling procedure and trying to have all states represented, all officially accredited TB diagnostic laboratories were requested to provide 15 isolates of $M$. bovis from every State available and the laboratory sheet with all relevant epidemiological information. To increase the sample and to cover the unrepresented or poorly represented States suspicious lesions of tuberculosis were obtained at slaughterhouses for culture and isolation: 269 isolates were obtained from laboratory and 609 from TB suspicious lesions.

\section{Laboratory analysis}

Samples from accredited laboratories were obtained by culturing in stonebrink or lowenstein-Jensen, and those from suspicious lesions in stonebrink. All isolates were genetically analyzed by-spoligotyping according to Kamerbeek et al(16), using the ISOGEN Maarssen kit (Netherlands). This method is based on the presence or absence of variants on 
en rastro para el cultivo y aislamiento de la bacteria: 269 aislados se obtuvieron en el muestreo pasivo y 609 en el muestreo activo.

\section{Análisis de laboratorio}

En el caso de las muestras de laboratorios, los aislados se obtuvieron por cultivo en medio de Stonebrink o Lowenstein-Jensen, y en el caso de lesiones obtenidas en el muestreo activo, los aislados se obtuvieron por cultivo en Stonebrink. Todos los aislados se analizaron genéticamente por el método de spoligotyping de acuerdo a Kamerbeek et al ${ }^{(16)}$, utilizando el kit de ISOGEN (Maarssen, Países Bajos). Este método se basa en la presencia o ausencia de variantes en las regiones variables (DVR, por sus siglas en inglés) de secuencias directas repetidas (DR, por sus siglas en inglés). Las DVR se componen de una secuencia repetida de $36 \mathrm{pb}$ conservada y una secuencia espaciadora variable de 35 a 41 pb. Por lo tanto, el blanco de la tipificación es la región variable(25).

\section{Análisis molecular}

Los patrones moleculares (espoligotipos) fueron convertidos a datos binarios de acuerdo a la presencia (1) o ausencia (0) de cada uno de los 43 espaciadores. Con apoyo de Excel los espoligotipos fueron organizados de acuerdo a su similitud genética. A todos aquéllos con espoligotipo idéntico se les asignó un número único, el cual se utilizó para realizar un análisis de clusters en SPSS ver. 16. Del resultado del análisis de clusters general se tomaron aquellos espoligotipos idénticos con dos o más aislados, para, por apreciación visual primero y por medio de un análisis de clusters parcial después, organizar grupos de similitud genética. Posteriormente, por medio de un análisis de frecuencia los espoligotipos se relacionaron con origen (Estado), sexo, raza y fin zootécnico de los animales afectados. Con la información relacionada al origen del animal (localidad), y con el apoyo de GoogleEarth se determinaron las coordenadas espaciales para cada uno de los aislados. the diverse variable regions (DVR) of repeated direct sequences (DR). The DVR consist of conserved repeated sequences of $36 \mathrm{bp}$ and variable spacer sequences of 35 to $41 \mathrm{bp}$ long. Therefore, the target sequence is the variable region ${ }^{(25)}$.

\section{Molecular analysis}

Molecular patterns (spoligotypes) were converted to binary data by the presence (1) or absence (0) of each of the 43 spacers. Spoligotypes were

Cuadro 1. Frecuencia de aislados de $M$. bovis obtenidos del ganado entre 2009 y 2010 en diferentes Estados de México

Table 1. Frequency of $M$. bovis isolates from cattle obtained between 2009 and 2010 in different States of Mexico

\begin{tabular}{lcc}
\hline State & Frequency & Proportion \\
\hline Jalisco & 153 & 16 \\
Edo de México & 110 & 12 \\
Hidalgo & 106 & 11 \\
Aguascalientes & 78 & 8 \\
Querétaro & 71 & 8 \\
Baja California & 55 & 6 \\
Guanajuato & 51 & 6 \\
Coahuila & 48 & 5 \\
Nayarit & 23 & 2 \\
San Luis Potosí & 21 & 2 \\
Veracruz & 19 & 2 \\
Zacatecas & 17 & 2 \\
Chihuahua & 15 & 2 \\
Guerrero & 15 & 2 \\
Michoacán & 15 & 2 \\
Durango & 14 & 2 \\
Morelos & 13 & 1 \\
Puebla & 13 & 1 \\
Sinaloa & 11 & 1 \\
Colima & 6 & 0.5 \\
Sonora & 5 & 0.5 \\
Campeche & 3 & 0.3 \\
Chiapas & 3 & 0.3 \\
Nuevo León & 2 & 0.2 \\
Oaxaca & 1 & 0.1 \\
Tlaxcala & 1 & 0.1 \\
TOTAL & 878 & \\
\hline & &
\end{tabular}




\section{RESULTADOS}

En el Cuadro 1 se muestra el estado de origen de los 878 aislados de M. bovis analizados. De un total de 26, los Estados que mayor cobertura tuvieron en términos de número de aislados fueron Jalisco ( $\mathrm{n}=153)$, el Estado de México $(\mathrm{n}=110)$, Hidalgo (Tizayuca, $n=106)$, Aguascalientes $(n=78)$, Querétaro $(n=71)$, Baja California $(n=55)$, Guanajuato $(n=51)$ y Coahuila $(n=48)$; el resto tuvo entre 23 y 1 aislados. El $66 \%(n=585)$ provinieron de ganado especializado en leche, el $7.5 \%$ fueron de cruzas de otras razas con ganado especializado en leche, el $4 \%$ de ganado especializado en carne y del $21 \%$ no se obtuvo información (Cuadro 2).

A partir de los 878 aislados se obtuvieron 194 espoligotipos, 45 con dos o más aislados y 149 con uno solo. Setenta y dos por ciento (72\%) de los espoligotipos agruparon en nueve clusters y $27 \%$ de los aislados cayeron dentro de dos espoligotipos SP1 $(n=107)$ y SP2 $(n=135)$ (Cuadro 3). En este cuadro se muestran los espoligotipos que incluyeron dos o más aislados con su respectivo patrón molecular, y se puede observar que los espoligotipos SP1 y SP2 fueron por mucho los de mayor frecuencia y cobertura, ya que incluyeron al mayor número de aislados y su presencia se observó en 18 y 20 Estados, respectivamente, de un total de 26 representados. La información de este cuadro se complementa con la Figura 1, donde se muestran los grupos (A-I) de similitud genética. El análisis de clusters indica una alta similitud de espoligotipos dentro de los grupos, lo que sugiere que la mayoría de los espoligotipos son derivados de los espoligotipos de mayor frecuencia en cada uno de los grupos. Aunque los espoligotipos SP1 y SP2, están presentes en la mayoría de los Estados, su presencia es especialmente notoria en aquéllos tradicionalmente especializados en la producción de leche: Aguascalientes ( $\mathrm{n}=9$ y 9, respectivamente), Coahuila ( $\mathrm{n}=4$ y 16), Estado de México $(\mathrm{n}=15$ y 17), Hidalgo ( $n=9$ y 16), Guanajuato $(n=7$ y 11$)$, Jalisco ( $n=20$ y 27), y Querétaro ( $n=4$ y 14). En el caso de Coahuila, la diferencia en la frecuencia entre SP1 y SP2 es notoria, el $33 \%$ de los aislados de ese estado pertenecen al SP2. grouped by genetic similarity in Excell. All isolates with identical spoligotype were identified with the same number, which was later used to perform a cluster analysis in SPSS.

Spoligotypes were later related to origin (state and county), sex, breed and productive performance of affected animals. Information about origin was used to obtain spatial coordinates of the herds with GoogleEarth.

\section{RESULTS}

The origin of the $878 \mathrm{M}$. bovis isolates by state is shown in Table 1. A total of 26 states were represented, those with large numbers of isolates were Jalisco $(n=153)$, the State of Mexico $(n=110)$, Hidalgo (Tizayuca, $n=106)$, Aguascalientes $(n=78)$, Querétaro $(n=71)$, Baja California $(n=55)$,

Cuadro 2. Spoligotypes más frecuentes de $M$. bovis obtenido del ganado entre 2009 y 2010 en México de acuerdo al tipo de producción del animal afectado

Table 2. Most frequent spoligotypes of $M$. bovis from cattle obtained between 2009 and 2010 in Mexico by type of production

\begin{tabular}{lccccc}
\hline Spoligotype & \multicolumn{4}{c}{ Production } & Total \\
\cline { 2 - 5 } & Beef & Dairy (HF) & Cross & INA & \\
\hline SP2 & 9 & 99 & 12 & 14 & 135 \\
SP1 & 4 & 61 & 11 & 28 & 107 \\
SP4 & 0 & 59 & 2 & 4 & 65 \\
SP7 & 2 & 43 & 5 & 6 & 59 \\
SP8 & 5 & 39 & 4 & 6 & 55 \\
SP12 & 1 & 44 & 2 & 2 & 49 \\
SP3 & 2 & 16 & 2 & 14 & 34 \\
SP6 & 2 & 9 & 0 & 16 & 32 \\
SP5 & 0 & 6 & 1 & 20 & 30 \\
SP11 & 1 & 13 & 2 & 3 & 19 \\
SP9 & 1 & 10 & 0 & 0 & 12 \\
SP33 & 0 & 4 & 0 & 7 & 11 \\
SP25 & 0 & 10 & 0 & 0 & 10 \\
SP35 & 0 & 10 & 0 & 0 & 10 \\
SP10 & 1 & 5 & 66 & 144 & 878 \\
\hline
\end{tabular}

$\mathrm{HF}=$ Holstein-Friesian, INA=Information not available. 
Feliciano Milián Suazo, et al. / Rev Mex Cienc Pecu 2012;3(4):459-471

Cuadro 3. Frecuencia de los spoligotypes más frecuentes de M. bovis obtenidos entre 2009 y 2010 en diferentes regiones de México, agrupados (A-I) por similitud genética

Table 3. Frequency of the most common spoligotypes of $M$. bovis from cattle obtained between 2009 and 2010 in different regions of Mexico, grouped (A-I) by genetic similarity

\begin{tabular}{|c|c|c|c|c|c|}
\hline Group & Spoligotype & Molecular pattern & Freq & $\%$ & Location \\
\hline \multirow[t]{13}{*}{ A } & SP24 & 0001101000001100111111111111111111111100000 & 3 & \multirow[t]{13}{*}{39} & $\mathrm{Hgo}(2)$, Qro \\
\hline & SP20 & 000100100000110011111111111111111111111100000 & 2 & & Ags, Dgo \\
\hline & SP25 & 00011010000011101111111111111111111111100000 & 10 & & $\operatorname{EdMX(3),~Gto,~Hgo(3),~Qro,~SLP(2)~}$ \\
\hline & SP2 & 0101101000001110111111111111111111111100000 & 135 & & $\begin{array}{l}\text { Ags(9), BC(3), Chiaps, Coah(16), Col, Dgo, EdMX(17), Gto(11), Gro, } \\
\text { Hgo(17), Jal(27), Mich, Mor(7), Qro(14), SLP, Sin(2), Ver, Zac(5) }\end{array}$ \\
\hline & SP29 & 0101101000101110111111111111111111111100000 & 2 & & Qro(2) \\
\hline & SP16 & 0101101000001110111111111111111111110100000 & 2 & & Coah, Gto \\
\hline & SP70 & 01011011000011101111111111111111111111100000 & 3 & & Ags, Gto, Jal \\
\hline & SP28 & 010110100001111011111111111111111111111100000 & 5 & & Hgo (3), Qro, EdMX \\
\hline & SP17 & 010110100000111001111111111111111111111100000 & 5 & & Coah (4), Jal \\
\hline & SP6 & 1101101000001100111111111111111111111100000 & 32 & & $\begin{array}{l}\text { Ags(3), Camp(3), Coah, Col, Dgo, EdMX(2), Gto, Gro, Hgo(3), jal(8), } \\
\text { Nay, Pue(2), SLP, Sin, Ver, desc (2). }\end{array}$ \\
\hline & SP8 & 1101101000001110111111111111111111111100000 & 55 & & $\begin{array}{l}\operatorname{Ags}(10), \operatorname{Coah}(2), \operatorname{EdMX}(5), \operatorname{Gto}(5), \operatorname{Hgo}(9), \operatorname{Jal}(13), \operatorname{Mich}(4), \text { Mor, } \\
\text { Qro(2), Zac(4). }\end{array}$ \\
\hline & SP1 & 0101101000001100111111111111111111111100000 & 107 & & $\begin{array}{l}\text { Ags(9), BC, Chih(6), Coah(4), Col, Dgo(3), EdMX(15), Gto(7), Gro(3), Hgo(9) } \\
\text { Jal(20), Mich, Nay(8), NL, Pue(5), Qro(4), SLP(5), Son, Tlax, Zac(3). }\end{array}$ \\
\hline & SP26 & 0101100000001100111111111111111111111100000 & 3 & & Col, Nay, Sin \\
\hline \multirow[t]{9}{*}{ B } & SP37 & 1101000000001110111111111111111111111100000 & 3 & \multirow[t]{9}{*}{15} & $\mathrm{Hgo}(2), \mathrm{BC}$ \\
\hline & SP36 & 110100000000110011111111111111111111111100000 & 2 & & Hgo, Jal \\
\hline & SP3 & 1101000000000000111111111111111111111100000 & 35 & & $\begin{array}{l}\text { Ags, BC(10), Chih, Dgo, EdMX(2), Gto(2), Jal(5), Mor, Nay(4), } \\
\text { Qro(4), Sin, Son, Ver, Zac. }\end{array}$ \\
\hline & SP34 & 1101000000000000111111111111111111101100000 & 2 & & EdMX, Jal \\
\hline & SP4 & 1101000000000010111111111111111111111100000 & 65 & & $\begin{array}{l}\operatorname{Ags}(4), \operatorname{BC}(14), \operatorname{EdMX}(16), \operatorname{Gto}(4), \operatorname{Hgo}(5), \operatorname{Jal}(10), \text { Nay, Qro(6), } \\
\operatorname{Sin}(2), \operatorname{Ver}(2), Z \mathrm{ZCC}\end{array}$ \\
\hline & SP39 & 11010010000000101111111111111111111111100000 & 3 & & $\mathrm{BC}(2)$, Gro \\
\hline & SP35 & 110100000000011011111111111111111111111100000 & 10 & & $\mathrm{BC}(2), \operatorname{EdMX}(4), \mathrm{Hgo}(2)$, Jal, Oro \\
\hline & SP99 & 1101000000000010110111111111111111111100000 & 6 & & Ags, $B C(2), \operatorname{EdMX}(2)$, AN5 \\
\hline & SP33 & 1101000000000000110111111111111111111100000 & 11 & & $\mathrm{BC}(8)$, Nay, EdMX, Desc. \\
\hline \multirow[t]{6}{*}{ C } & SP7 & 1101101000001110111111111111110000001100000 & 62 & \multirow[t]{6}{*}{5} & $\begin{array}{l}\operatorname{Ags}(6), B C, C h i a p s, \operatorname{EdMx}(10), \operatorname{Gto}(9), \operatorname{Gro}, \operatorname{Hgo}(8), \operatorname{Jal}(7), \operatorname{Mich}(2), \\
\text { Mor, Nay, Oax, Qro(7), SLP(3), Son, } \operatorname{Ver}(2)\end{array}$ \\
\hline & SP42 & 1101101000001010111111111111110000001100000 & 2 & & Ags, Gro \\
\hline & SP46 & 1101101000001110111111111111100000001100000 & 4 & & Ags (3), Jal, \\
\hline & SP45 & 1101101000001110111111111110110000001100000 & 3 & & EdMX, Gto, Qro \\
\hline & SP43 & 1101101000001100111111111110110000001100000 & 2 & & EdMX, Qro \\
\hline & SP5 & 1101101000001100111111111111110000001100000 & 30 & & $\begin{array}{l}\text { Ags(3),Dgo, EdMX, Gro, Jal(5), Mich, Nay, Pue(2), Qro, SLP(3), Sin, } \\
\text { Ver(8), Zac, Desc }\end{array}$ \\
\hline \multirow[t]{4}{*}{ D } & SP12 & 11011111011111101111011111111111111111100000 & 49 & \multirow[t]{4}{*}{8} & Ags, BC, Coah(2), EdMX(3), $\operatorname{Hgo}(20), \operatorname{Jal}(9), \operatorname{Mich}(3)$, Nay, Qro(9) \\
\hline & SP32 & 1001111101111110111101111111111111111100000 & 3 & & $\mathrm{Hgo}(3)$ \\
\hline & SP11 & 1101111101111100111101111111111111111100000 & 19 & & Chih(3), Coah(2), Col, Dgo, Gto, Hgo(3), Jal(6), NL, Sin \\
\hline & SP13 & 1101111101111100111111111111111111111100000 & 5 & & Chih, Dgo, Qro, BCG(2) \\
\hline \multirow[t]{2}{*}{ E } & $\mathrm{SP} 30$ & 1000000000000000000001111111111111111100000 & 3 & \multirow[t]{2}{*}{0.5} & EdMX, Jal, Qro \\
\hline & SP31 & 1000000000000000100001111111111111111100000 & 2 & & Coah, Jal \\
\hline \multirow[t]{2}{*}{$\mathrm{F}$} & SP10 & 1101111101011110111111111111111111111100000 & 7 & \multirow[t]{2}{*}{1.1} & Coah, Gro, Hgo, Jal, Mich, Mor, Qro \\
\hline & SP41 & 11010111010111101111111111111111111111100000 & 3 & & Hgo(2), Qro \\
\hline \multirow[t]{2}{*}{ G } & SP15 & 0001111001011110111111111110001111111100000 & 3 & \multirow[t]{2}{*}{0.5} & EdMX, Gto, Jal \\
\hline & SP14 & 0001111001011100111111111110001111111100000 & 2 & & Dgo, Jal \\
\hline \multirow[t]{2}{*}{ H } & SP9 & 1101111101011110111111111110001111111100000 & 12 & \multirow[t]{2}{*}{1.7} & Ags (8), Coah, EdMX(2), Hgo \\
\hline & SP40 & 1101011101001100111110111111111111111100000 & 4 & & AN5(3), Coah \\
\hline & SP38 & 11010001001000101111111111111111111111100000 & 2 & \multirow[t]{4}{*}{1.1} & EdMX, Qro \\
\hline & SP58 & 010110100000110011100001111111111111111100000 & 2 & & Jal, Ags \\
\hline & SP49 & 11011011010000001111011111111111111111100000 & 3 & & Coah(2), Gto \\
\hline & SP44 & 1101101000001110111000000000000001111100000 & 4 & & Ags, Jal, Mich(carne), Qro \\
\hline & SUB-TOTAL & & 729 & 72 & \\
\hline & Single Spolig & types & 149 & 17 & \\
\hline & TOTAL & & 878 & & \\
\hline
\end{tabular}


Aunque en general se observa una gran diversidad regional de espoligotipos, en algunos de ellos se observa cierta regionalización (Cuadro 4). Por ejemplo, a pesar de que los espoligotipos SP1 y SP2 fueron los más frecuentes y los de mayor distribución en el País, en la región de Tijuana, BC tuvieron poca presencia $(n=3)$. En esta región los espoligotipos más frecuentes fueron SP3 $(n=10)$, SP4 $(n=14)$ y SP33 $(n=8)$, los cuales agrupan en el

Figura 1. Dendrograma que muestra la similitud/diferencia genética de los spoligotypes de aislados de $M$. bovis obtenidos entre 2009 y 2010 del ganado en diferentes regiones de México

Figure 1. Dendrogram showing the genetic similarity/difference of the spoligotypes of $M$. bovis obtained between 2009 and 2010 from cattle in different regions of Mexico

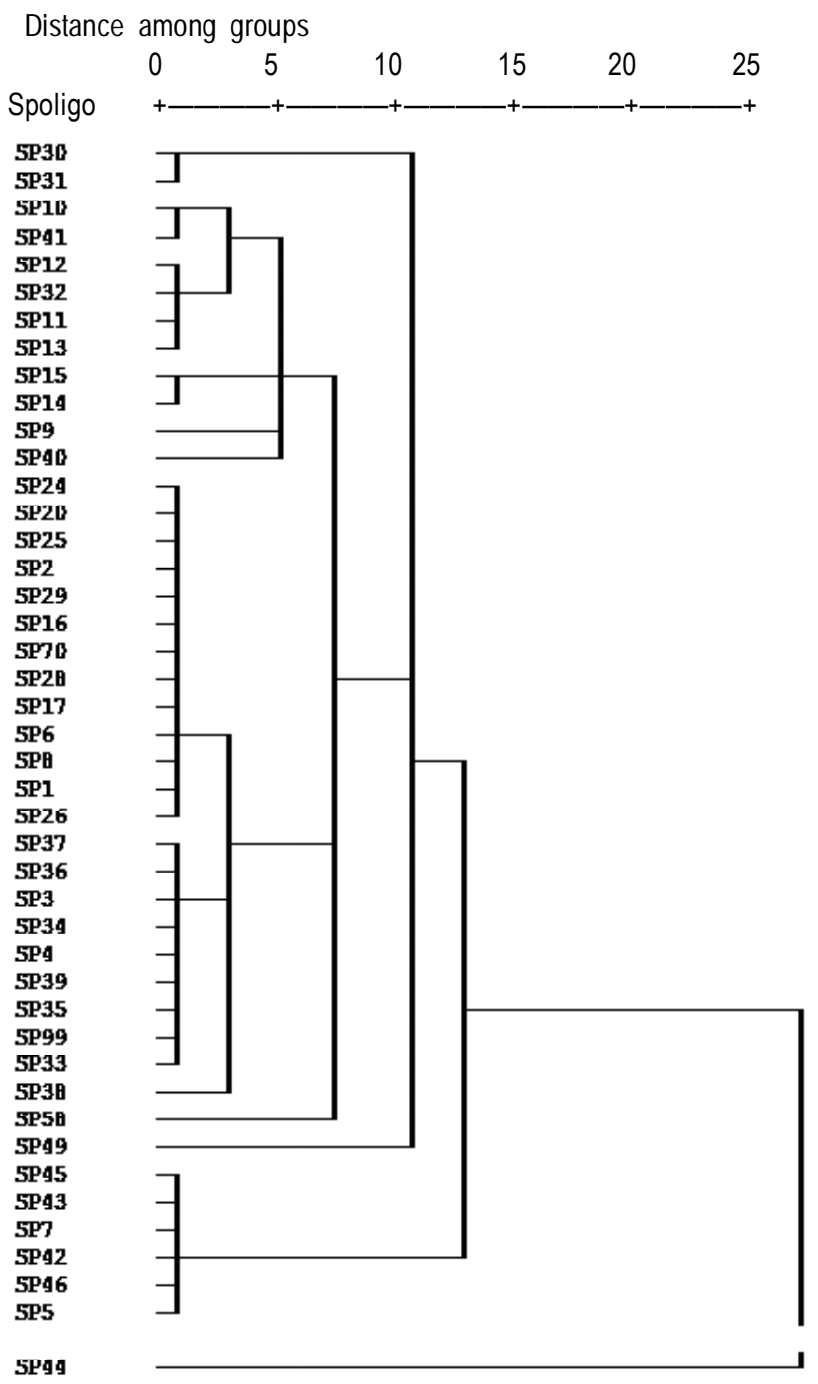

Guanajuato $(n=51)$ and Coahuila $(n=48)$; the rest had between 1 and 23 isolates. Sixty six percent of the isolates $(n=585)$ came from dairy cattle (Holstein), $7.5 \%$ from crossbreeds with Holstein, $4 \%$ beef cattle, and $21 \%$ with no information about breed (Table 2).

From the isolates total, 194 different spoligotypes were obtained, 45 grouping two or more isolates and 149 orphans. Seventy two percent of the spoligotypes were grouped in just nine clusters and $27 \%$ of the isolates fell into two spoligotypes SP1 $(n=107)$ and SP2 $(n=135)$ (Table 3). This table

Cuadro 4. Frecuencia de los spoligotypes más comunes de $M$. bovis obtenidos del ganado entre 2009 y 2010 en diferentes regiones de México, por estado

Table 4. Frequency of the most common spoligotypes of $M$. bovis from cattle obtained between 2009 and 2010 in different regions of Mexico

\begin{tabular}{|c|c|c|c|c|c|c|c|c|c|c|c|c|c|}
\hline \multirow[b]{2}{*}{ State } & \multicolumn{12}{|c|}{ Spoligotype } & \multirow[b]{2}{*}{ Total } \\
\hline & 1 & 2 & 3 & 4 & 5 & 6 & 7 & 8 & 9 & 11 & 12 & 33 & \\
\hline Aguascalientes & 9 & 9 & 1 & 4 & 3 & 3 & 6 & 10 & 8 & & 1 & & 78 \\
\hline B. California & 1 & 3 & 10 & 14 & & & 1 & & & & & 8 & 55 \\
\hline Campeche & & & & & & 3 & & & & & & & 3 \\
\hline Chiapas & & 1 & & & & & 1 & & & & & & 2 \\
\hline Chihuahua & 6 & & 1 & & & & & & & 3 & & & 15 \\
\hline Coahuila & 4 & 16 & & & & 1 & & 2 & 1 & 2 & 2 & & 48 \\
\hline Colima & 1 & 1 & & & & 1 & & & & 1 & & & 6 \\
\hline Durango & 3 & 1 & 1 & & 1 & 1 & & & & 1 & & & 14 \\
\hline Edo de México & 15 & 17 & 2 & 16 & 1 & 2 & 10 & 5 & 2 & & 3 & 1 & 110 \\
\hline Guanajuato & 7 & 11 & 1 & 4 & & 1 & 10 & 5 & & 1 & & & 51 \\
\hline Guerrero & 3 & 1 & & & 1 & 1 & 1 & & & & & & 15 \\
\hline Hidalgo & 9 & 16 & & 5 & & 3 & 8 & 9 & 1 & 3 & 20 & & 106 \\
\hline Jalisco & 20 & 27 & 5 & 10 & 5 & 8 & 7 & 13 & & 6 & 9 & & 153 \\
\hline Michoacán & 1 & 1 & & & 1 & & 2 & 4 & & & 3 & & 15 \\
\hline Morelos & & 7 & 1 & & & & 1 & 1 & & & & & 33 \\
\hline Nayarit & 8 & & 4 & 1 & 1 & 1 & 1 & & & & 1 & 1 & 23 \\
\hline Nuevo León & 1 & & & & & & & & & 1 & & & 2 \\
\hline Oaxaca & & & & & & & 1 & & & & & & 1 \\
\hline Puebla & 4 & & & & 2 & 2 & & & & & & & 13 \\
\hline Querétaro & 4 & 14 & 4 & 6 & 1 & & 4 & 2 & & & 9 & & 71 \\
\hline S L Potosí & 5 & 1 & & & 3 & 1 & 3 & & & & & & 21 \\
\hline Sinaloa & & 2 & 1 & 2 & 1 & 1 & & & & 1 & & & 11 \\
\hline Sonora & 1 & & 1 & & & & 1 & & & & & & 5 \\
\hline Tlaxcala & 1 & & & & & & & & & & & & 1 \\
\hline Veracruz & & 1 & 1 & 2 & 8 & 1 & 2 & & & & & & 19 \\
\hline Zacatecas & 3 & 5 & 1 & 1 & 1 & & & 4 & & & & & 17 \\
\hline TOTAL & 107 & 135 & 34 & 65 & 30 & 32 & 59 & 55 & 12 & 19 & 49 & 11 & 878 \\
\hline
\end{tabular}


grupo B, sugiriendo parentesco de cepas. SP3 y SP33 fueron casi exclusivos de la región. En el caso de Chihuahua, sólo se observó presencia de SP1. Para Nayarit y Morelos, dos estados no considerados especializados en producción de leche, tuvieron la presencia de SP1 $(n=8)$ y SP2 $(n=7)$, donde sólo un aislado fue de ganado productor de carne, por lo que se presume que el resto provienen de ganado productor de leche o de sus cruzas. En Zacatecas se observó la presencia de SP1 $(n=3)$ y SP2 ( $n=5)$, seis de estos fueron obtenidos de ganado para carne, por lo que se supone contacto estrecho entre razas en esta región. Situación similar se presentó en Guanajuato, donde siete aislados fueron de ganado para carne, los cuales comparten espoligotipo con aislados de ganado para leche. En Aguascalientes, además de SP1 y SP2 se nota la frecuencia de SP8 y SP9, donde SP8 está emparentado con SP1 y SP2, ya que están en el mismo grupo; sin embargo, SP9 pertenece a un grupo diferente. En la región de La Laguna (Coahuila y Durango), los espoligotipos de mayor frecuencia fueron SP1 y SP2; no obstante, otros espoligotipos de alta frecuencia en otras zonas lecheras del País, tales como SP4, SP7 y SP8, estuvieron ausentes. En el caso de Chihuahua, sólo dos espoligotipos predominan, SP1 y SP11, los cuales son muy diferentes entre sí genéticamente, lo que sugiere dos fuentes grandes de infección y poca entrada de animales de otras regiones.

\section{DISCUSIÓN}

A partir de 878 aislados de $M$. bovis obtenidos del ganado en diferentes regiones de México se generaron 197 espoligotipos, 45 con dos o más aislados y 149 con sólo uno. Los espoligotipos más frecuentes fueron SP1 y SP2, presentes en la mayoría de los estados representados, en especial en aquéllos tradicionalmente enfocados a la producción de leche. La mayoría de los espoligotipos que involucran a un solo aislado son muy parecidos a los espoligotipos de mayor frecuencia en la muestra, la diferencia en la presencia/ausencia de espaciadores en la mayoría de los casos es de sólo uno, lo que sugiere que shows the molecular pattern of spoligotypes involving two or more isolates, spoligotypes SP1 and SP2 were by far the ones with the largest number of isolates observed in 18 and 20 respectively, of a total of 26 states represented. A dendrogram showing the genetic relationship of isolates is presented in Figure 1, where a high similarity of spoligotypes is observed, suggesting that most spoligotypes derived from those with higher frequency. Although spoligotypes SP1 and $\mathrm{SP} 2$, are present in most states, their presence is especially high in states with high population of dairy cattle: Aguascalientes $(\mathrm{n}=9$ and 9, respectively), Coahuila ( $\mathrm{n}=4$ and 16), the state of Mexico $(n=15$ and 17), Hidalgo $(n=9$ and 16), Guanajuato ( $\mathrm{n}=7$ and 11), Jalisco ( $\mathrm{n}=20$ and 27), and Queretaro ( $\mathrm{n}=4$ and 14). In the case of Coahuila, the difference in frequency between SP1 and SP2 is notorious, $33 \%$ of the total isolates in the state belong to SP2.

Although in general there is a high diversity of spoligotypes in Mexico, some show some kind of

Figura 2. Distiribución espacial de los spoligotypes de mayor frecuencia de aislados de $M$. bovis obtenidos del ganado en diferentes regiones de México

Figure 2. Space distribution of the most frequent $M$. bovis spoligotypes-from of $M$. bovis from cattle obtained between 2009 and 2010 in different regions of Mexico

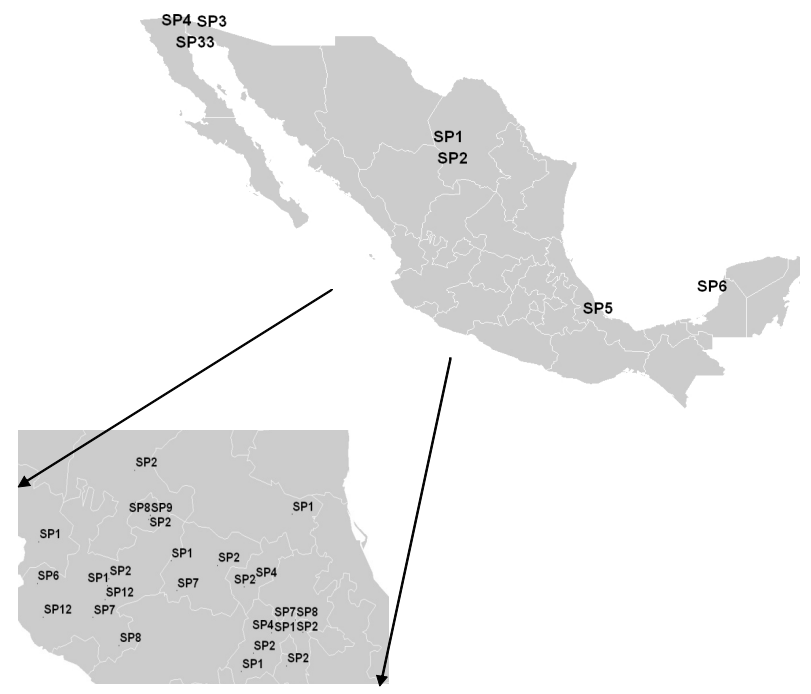


estos son el resultado de nuevas líneas genéticas derivadas de mutaciones no letales en infecciones recientes.

La distribución de un mismo espoligotipo en diferentes regiones puede ser un indicativo de su alta movilización de ganado. De acuerdo a la frecuencia de los espoligotipos, esta movilización es más frecuente entre cuencas lecheras del centro del país y la región de La Laguna. Las cuencas del norte, Chihuahua y Baja California, parecen tener menos intercambio de animales, lo cual puede ser una consecuencia lógica de la distancia geográfica. La zona de Baja California tiene tres espoligotipos que la diferencian del resto, SP3, SP4 y SP33, los cuales están altamente emparentados, ya que sólo existe un espaciador de diferencia entre ellos, lo que sugiere poca entrada de animales de otras regiones de México. El caso de Hidalgo es muy particular, y que casi todos los aislados provienen de Tizayuca, un complejo lechero con alta densidad de población, donde se esperaría predominancia de algún espoligotipo en particular; no obstante en la base de datos, se observó la presencia de casi todos los de mayor regionalization (Table 4). For example, while spoligotypes SP1 and SP2 were the most frequent and have the largest distribution in the country, in Tijuana, BC are rare $(n=3)$. In this region the most frequent spoligotypes are SP3 $(n=10)$, SP4 $(n=14)$ and SP33 $(n=8)$, which group in cluster $B$, suggesting genetic relationship between this. Spoligotypes SP3 and SP33 were almost exclusive of this region. In Chihuahua, only SP1 was observed but not SP2. For Nayarit and Morelos, two states not considered specialized in milk production, had SP1 $(n=8)$ and SP2 $(n=7)$, however, only one isolate came from beef cattle, we believe the rest came from dairy cattle. SP1 $(n=3)$ and SP2 $(n=5)$ were observed in Zacatecas, six of these were obtained from beef cattle, transmission of BTB between breeds in this region is suspected. A similar situation was observed in Guanajuato, where seven isolates came from beef cattle, suggesting, again, transmission between breeds. In Aguascalientes, in addition to SP1 and SP2 the frequency of SP8 and SP9 is notorious. The molecular pattern of SP8 is quite similar to the one in SP1 and SP2, as they are in the same group; however, SP9 belong to a different group. In La Laguna (Coahuila and

Cuadro 5. Spoligotypes de $M$. bovis encontrados en razas especializadas en carne que también fueron encontrados en razas especializadas en leche en diferentes regiones de México

Table 5. Spoligotypes of M. bovis found in beef and dairy breeds in different regions of Mexico

\begin{tabular}{|c|c|c|c|c|c|c|}
\hline \multirow[b]{2}{*}{ Spoligotype } & & \multicolumn{5}{|c|}{ Type of producción } \\
\hline & & Beef & & Dairy (HF) & HF Cross & Total \\
\hline SP2 & 0101101000001110111111111111111111111100000 & 9 & $\begin{array}{l}\text { Chiaps-PichucalcoDgo-TepehuanesGto(2)-SF } \\
\text { Rincón,SFpeJal-Valle GpeZac-Cañitas(3), Fres(1) }\end{array}$ & 99 & 12 & 135 \\
\hline SP1 & 0101101000001100111111111111111111111100000 & 4 & Gto(2)-SFRincón, LeónZac(2)-Morelos, Fresnil & 61 & 11 & 107 \\
\hline SP7 & 11011010000011101111111111111110000001100000 & 2 & Gto-León, La lomaMich-Coahuayana & 43 & 5 & 59 \\
\hline SP8 & 1101101000001110111111111111111111111100000 & 5 & $\begin{array}{l}\text { Gto-León-Los ArcosJal-Tepa-LagunillasMich- } \\
\text { Huetamo, ParoZac (2)-TeulGlez, SFRin }\end{array}$ & 39 & 4 & 55 \\
\hline SP12 & 1101111101111110111101111111111111111100000 & 1 & Mich-M. castellanos & 44 & 2 & 49 \\
\hline SP3 & 11010000000000001111111111111111111111100000 & 2 & Jal-Tepa, El LavaderoJal-Tepa, El Lavadero & 16 & 2 & 34 \\
\hline SP6 & 1101101000001100111111111111111111111100000 & 2 & Dgo-El Oro, Sta MaGto-León, Los Arcos & 9 & 0 & 32 \\
\hline SP11 & 1101111101111100111101111111111111111100000 & 1 & Gto-León, Sta Rosa & 13 & 2 & 19 \\
\hline SP9 & 1101111101011110111111111110001111111100000 & 1 & EdoMX-Tejupilco, Zacat & 10 & 0 & 12 \\
\hline SP10 & 1101111101011110111111111111111111111100000 & 1 & Gro-Jaleaca de Catalán & 5 & 0 & 7 \\
\hline Total & & 28 & & 339 & 38 & 878 \\
\hline
\end{tabular}

$\mathrm{HP}=$ Holstein Friesian 
frecuencia, si bien resalta el SP12, observado también con frecuencia en Jalisco y Querétaro, lo que sugiere intercambio frecuente de animales entre Tizayuca y estos dos estados.

Varios de los espoligotipos encontrados en aislados de ganado para leche fueron también encontrados en aislados de ganado para carne y en aislados de Estados no especializados en producción de leche. Esto fue notorio en los estados de Zacatecas, Guanajuato y Morelos, lo que sugiere transmisión entre razas. Desde luego, en el caso de otros Estados no puede descartarse que se trate de ganado para leche, pero desafortunadamente las muestras no tenían información sobre la raza del animal afectado en todos los casos para confirmar este dato.

Nuestros resultados coinciden con lo reportado con anterioridad sobre diversidad genética de espoligotipos de $M$. bovis por región en México(22,26,27). Los espoligotipos SP2, SP8, SP9, SP12 y SP17 ya habían sido señalados en hatos lecheros de Ciudad Juárez, Chihuahua(22); sin embargo, espoligotipos clasificados en www.mbovis.org como SB0987, SB0272, SB0985, SB0327 y SB0807 reportados en ese mismo estudio no fueron encontrados en nuestra base de datos, lo que sugiere que estos espoligotipos pueden ser particulares de esa región del País. Una ventaja de nuestro estudio en relación a trabajos previos es el alto número de aislados incluido y el número de estados representados, lo que garantiza una mayor representatividad de aislados por región y permite conclusiones más sólidas sobre la regionalización de algunos espoligotipos.

El la mayoría de los estudios de epidemiología molecular donde se involucra una cantidad indeterminada de aislados de $M$. bovis para determinar su distribución regional, se carece de representatividad en el muestreo. Esto es, el número de aislados analizados es más bien consecuencia de la disponibilidad de los mismos, que de un tamaño de muestra probabilístico, nuestro estudio no es la excepción. Por lo tanto, es probable que la poca o nula presencia de algunos de los
Durango), SP1 and SP2 were also the most frequent spoligotypes; however, other spoligotypes of high frequency in other milk producing areas, such as SP4, SP7 and SP8 were absent. In the case of Chihuahua, two predominant spoligotypes, SP1 and SP11, which are quite different genetically, suggest different source of infection.

\section{DISCUSSION}

From $878 \mathrm{M}$. bovis isolates from cattle in different regions of Mexico, 197 spoligotypes were obtained, 45 with two or more isolates and 149 with only one. The most frequent spoligotypes were SP1 and SP2, present in almost all states represented, especially in those traditionally focused on milk production. Most single-isolate spoligotypes are quite similar to more frequent spoligotypes, in most cases only one spacer difference suggesting genetic evolution, perhaps as a consequence of non-lethal mutations.

The presence of the same spoligotype in different regions may be a consequence of the indiscriminate movement of animals between regions. According to frequency of spoligotypes, it seems that mobilization is frequent between central Mexico and La Laguna. Regions in the North, Chihuahua and Baja California, appear to have less exchange of animals, which may be a logical consequence of the geographic distance. The area of Baja California has three highly related spoligotypes quite different from spoligotypes in other regions, SP3, SP4 and SP33, suggesting little entry of animals from other regions. Hidalgo is a special place, almost all isolates came from Tizayuca, a milking-complex with a high density of dairy cattle where the same spoligotype would be expected; however, almost all spoligotypes were observed, especially SP12, observed also in Jalisco and Querétaro, suggesting frequent exchanges of animals between these three states.

Spoligotypes found in dairy cattle were also found in beef cattle This was notorious in Zacatecas, Guanajuato and Morelos, suggesting transmission 
espoligotipos en algunas regiones sea más bien consecuencia de la poca cantidad de aislados proporcionados, que de una ausencia real de los mismos. La sistematización en la tipificación (espoligotipificación) de todos los aislados de $M$. bovis obtenidos por los laboratorios regionales de diagnóstico ayudaría enormemente a determinar la posible regionalización de los espoligotipos del ganado en México.

Otra deficiencia de este tipo de estudios es la carencia de información epidemiológica disponible para cada aislado, lo que limita la validez de las conclusiones, y que se pueda explicar mejor el comportamiento del Mycobacterium en una determinada región geográfica. En el caso de México, existe un temor infundado por parte de autoridades de la Campaña de Tuberculosis de algunos Estados, de que la información se use para fines de "fiscalización", por lo que no proporcionan la información epidemiológica completa a pesar de contar con ella, lo que desde luego imposibilita el desarrollo de estudios epidemiológicos completos.

Es pertinente mencionar que spoligotyping no es un método de alto poder de discriminación, más bien se le considera un método apropiado para estudios globales para la identificación de grupos, los cuales deberán ser posteriormente analizados con un método más discriminatorio, como es el caso de VNTR (variable number tandem repeats, por sus siglas en inglés). De hecho se ha determinado por conveniencia internacional utilizar spoligotiping para la formación de grupos de aislados genéticamente relacionados y VNTR para definir cepas específicas y definir transmisión(28). En el caso de los aislados de este estudio, los análisis con VNTR están pendientes.

\section{CONCLUSIONES E IMPLICACIONES}

Se observa una gran diversidad de espoligotipos en las diferentes regiones de México, lo que puede ser consecuencia de la movilización frecuente de ganado. La mayoría de los espoligotipos de un solo aislado tienen una alta similitud con aquéllos de between breeds. Our results are consistent with previous reports about genetic diversity of $M$. bovis in Mexico(22,26,27). Spoligotypes SP2, SP8, SP9 SP12 and SP17 have been reported in Ciudad Juárez, Chihuahua(22); however, spoligotypes classified in www.mbovis.org as SB0987, SB0272, SB0985, SB0327 and SB0807 reported in the same study were not found in our database, suggesting that these spoligotypes may be particular of that region. An advantage of our study in relation to previous reports is the high number of isolates included and the number of states represented, which means higher representativity of isolates by region.

In most studies of molecular epidemiology, an undetermined number of isolates is used, usually those available at the time, therefore, representativity is lacking. That is, the number of isolates analyzed is rather a consequence of the availability, than a probabilistic sample, and our study is not the exception. Therefore the low or no presence of some of the spoligotypes in some regions could be the result of the low number of isolates provided rather than a lack of them in the population. The systematic typing of isolates in diagnostic laboratories would greatly help to identify regionalization of spoligotypes in cattle in Mexico.

One deficiency of this study is the lack of enough epidemiological information for each isolate, limiting the validity of the conclusions. In the case of Mexico, there is an unfounded fear by local authorities of the Tuberculosis Campaign, that information could be used for "punishment" purposes; therefore, only partial epidemiological information is provided.

It is important to mention that spoligotyping is not a method with high discrimination power, it is rather a good method for identification of large groups, which are later analyzed with more discriminatory methods. In fact there is an international agreement about using spoligotyping for general grouping of isolates, and then use VNTR to define strains and transmission(28). In our study, results from VNTR are pending. 
mayor frecuencia, por lo que se deduce que son consecuencia de mutaciones no letales que dan origen a nuevas líneas genéticas. La presencia de espoligotipos idénticos en ganado especializado en leche y ganado para carne, indica transmisión de tuberculosis de una raza de ganado a la otra, lo cual puede tener serias repercusiones epidemiológicas por razones de comercialización y de exportación de ganado. Aunque en este caso no se puede descartar que se trate de engordas familiares de ganado dentro de las explotaciones lecheras, en cuyo caso no representaría un riesgo para la ganadería de carne en su generalidad. Se concluye que una mayor representatividad de aislados de las diferentes regiones, una mayor representatividad de las diferentes razas de ganado y una mejor captura de información epidemiológica ayudaría a tener un diagnóstico de la situación de los espoligotipos de $M$. bovis en México más precisa. No obstante, ahora se cuenta con una base de datos importante que en mucho apoyará la toma de decisiones para el mejor control de la tuberculosis en México, y para mejorar los acuerdos de comercialización nacional e internacional de animales.

\section{AGRADECIMIENTOS}

Se agradece la participación de los Laboratorios Regionales de Diagnóstico de TB en México, que amablemente proporcionaron aislados de $M$. bovis para la realización de este trabajo.

\section{LITERATURA CITADA}

1. Bourne FJ. Bovine TB: The scientific evidence. Final report of the independent scientific group on cattle TB. Defra, UK. 2007.

2. Pollock M, Neill D. Mycobacterium bovis infection and tuberculosis in cattle. Vet J 2002;(2):115-27.

3. Gibson AL, Hewinson G, Goodchild T, Watt B, Story A, Inwald J, Drobniewski FA. Molecular epidemiology of disease due to Mycobacterium bovis in humans in the United Kingdom. J Clin Microbiol 2004;(42):431-434.

\section{CONCLUSIONS AND IMPLICATIONS}

There is a large diversity of spoligotypes in cattle in Mexico, which can be the result of the frequent movement of animals between regions. Most singleisolate spoligotype have a high genetic similarity with those of higher frequency, therefore, genetic evolution to non-lethal mutations given origin to new genetic lines is hypothesized. Presence of identical spoligotypes in dairy and beef cattle indicates TB transmission between population's breeds. This may have serious epidemiological implications for marketing and exportation of animals. It is possible, however, the existence of feedlots in dairy areas, in such a case, dairy do not represent a risk to beef cattle populations. It was concluded that a better representativity of isolates by region and breed of cattle, and also the collection of more epidemiological data would help in having a more accurate diagnosis of the distribution of $M$. bovis spoligotypes in Mexico. However, there is now an important database that will greatly support decision-making in controlling tuberculosis in Mexico and in supporting international agreements for exportation of animals and animal products.

\section{ACKNOWLEDGMENTS}

We would like to thank to all Regional TBDiagnostic Laboratories in Mexico which kindly provided $M$. bovis isolates for this study.

End of english version

4. Romero B, Aranaz A, de Juan L, Alvarez J, Bezos J, Mateos A, Gómez-Mampaso E, Domínguez L. Molecular epidemiology of multidrug-resistant Mycobacterium bovis isolates with the same profile as isolates from animals. J Clin Microbiol 2006;(449): 3405-3408.

5. Lari N, Rindi L, Bonanni D, Tortoli E, Garzelli C. Molecular analysis of clinical isolates of Mycobacterium bovis recovered from humans in Italy. J Clin Microbiol 2006;(4411):4218-4221.

6. de la Rua-Domenech R. Human Mycobacterium bovis infection in the UK: incidence, risks, control measures and review of 
the zoonotic aspects of bovine tuberculosis. Tuberc 2006;(86):77-109.

7. Pérez-Guerrero L, Milián SF, Arriaga DC, Romero TC, Escartín ChM. Epidemiología molecular de la tuberculosis bovina y humana en Querétaro, México. Rev Sal Púb Méx 2008;(50):16.

8. Rodwell TC, Moore M, Moser KS, Brodine SK, Strathdee SA. Tuberculosis from Mycobacterium bovis in binational communities, United States. Emerg Infect Dis 2008;(146):909916.

9. Milian-Suazo F, Harris B, Arriaga DC, Romero TC, Stuber T, Alvarez OG, Morales LA, et al. Molecular epidemiology of Mycobacterium bovis: usefulness in international trade. Prev Vet Med 2008;(87):261-271.

10. Plan Estratégico de la Campaña Nacional de la Tuberculosis Bovina en México, 2008-2012. Dirección General de Salud Animal. Campaña Nacional Contra la Tuberculosis Bovina. [en línea]. http://www. senasica.gob.mx/?id=801. Consultado Abr 15, 2011.

11. White PC, Bohm M, Marion G, Hutchings, MR. Control of bovine tuberculosis in British livestock: there is no 'silver bullet'. TIM 2008;420-427.

12. Green DM, Kiss IZ, Mitchell AP, KAO RR. Estimates for local and movement-based transmission of bovine tuberculosis in British cattle. Proc Royal Soc Biol Sci 2008;(275):1001-1005.

13. Ramirez-Villaescusa AM, Medley GF, Mason S, Green IE. Herd and individual animal risks associated with bovine tuberculosis skin test positivity in cattle in herds in south west England. Prev Vet Med 2009;(92):188-198.

14. Skuce RA, Mallon TR, McCormick CM, McBride SH, Clarke G, Thompson A, Couzens C, Gordon AW, McDowell SWJ. Mycobacterium bovis genotypes in Northern Ireland: herdlevel surveillance (2003 to 2008). Vet Rec 2010;(167);684689.

15. Durr PA, Clifton-Hadley RS, Hewinson RG. Molecular epidemiology of bovine tuberculosis II. Applications of genotyping. Rev Sci Tech Off Int Epiz 2000;(19):689-701.

16. Kamerbeek J, Schouls L, Kolk A, van Agterveld M, van Soolingen D, Kuijper S, Bunschoten A, Molhuizen H, Shaw R, Goyal M, van Embden J: Simultaneous detection and strain differentiation of Mycobacterium tuberculosis for diagnosis and epidemiology. J Clin Microbiol 1997;(35):907-914.

17. Collins DM, Gabric DM, de Lisle GW. Typing of Mycobacterium bovis isolates from cattle and other animals in the same locality. NZ Vet J 1988;(36):45-46.
18. van Soolingen D, de Haas PEW, Haagsma J, Eger T, Hermans PWM, Ritacco V, Alito A, van Embden JDA. Use of various genetic markers in differentiation of Mycobacterium bovis strains from animals and humans and for studying epidemiology of bovine tuberculosis. J Clin Microbiol 1994;(32):2425-2433.

19. Zumarraga MJ, Martin C, Samper S, Alito A, Latini O, Bigi F, Roxo E, Cicuta ME, Errico F, Ramos MC, van Soolingen D, Romano MI, Usefulness of spoligotyping in molecular epidemiology of Mycobacterium bovis-related infections in South America. J Clin Microbiol 1999;(372):296-303.

20. Gutiérrez M, Samper S, Gavigan JA, García Marín JF, Martín C. Differentiation by molecular typing of Mycobacterium bovis strains causing tuberculosis in cattle and goats. J Clin Microbiol 1995;(33):2953-2956.

21. Aranaz A, Liébana E, Mateos A, Dominguez L, Vidal D, Domingo M, Gonzolez O, et al. Spacer oligonucleotide typing of Mycobacterium bovis strains from cattle and other animals: a tool for studying the epidemiology of tuberculosis. J Clin Microbiol 1996;(34):2734-40.

22. Cobos-Marín L, Montes-Vargas J, Zumarraga M, Cataldi A, Romano MI, Estrada-Garcia I, Gonzalez-y-Merchand JA. Spoligotype analysis of Mycobacterium bovis isolates from Northern México. Can J Microbiol 2005;(51):996-1000.

23. Parra A, Larrasa J, García A, Alonso JM, de Mendoza JH. Molecular epidemiology of bovine tuberculosis in wild animals in Spain: a first approach to risk factor analysis. Vet Microbiol 2005;(110):293-300.

24. Amadio A, Romano MI, Bigi F, Etchechoury I, Kubica T, Niemann S, Cataldi A, Caimi K. Identification and characterization of genomic variations between Mycobacterium bovis and M. tuberculosis H37Rv. J Clin Microbiol 2005(435):2481-2484.

25. Acosta-Salinas R, Estrada-Chávez C, Milián-Suazo F. Tipificación de cepas de mycobacterium bovis: revisión. Tec Pecu Méx 2009;(47):389-412.

26. Milián SF, Banda RV, Ramírez CC, Arriaga DC. Genotyping of Mycobacterium bovis by geographic location within México. Prev Vet Med 2002;(55):255-264.

27. Santillan-Flores MA, Flores J, Arriaga-Diaz C, Romero-Torres C, Suárez-Güemes F, Espitia C. Polymorphism of the PE domain of PE/PE_PGRS sequences in clinical isolates of Mycobacterium bovis in Mexico. Vet Microbiol 2006;(115):364-369.

28. Smith NH, Kremer K, Inwald J, Dale J, Driscoll JR, Gordon SV, Van Soolingen D, Hewinson RG, Smith JM. Ecotypes of the Mycobacterium tuberculosis complex. J Theor Biol 2006;(239):220-225. 
